\title{
Reduced Burst Release from ePTFE Grafts: A New Coating Method for Controlled Drug Release
}

\author{
Hye Yeong Nam, Dae Joong Kim," Hyun Jung Lim, Byung Ha Lee, Insu Baek, Sang Hun Park, and Jong-sang Park \\ School of Chemistry \& Afolecular Engineering, Seoul National Lniversitvi, Seoul 151-742, Korea \\ "E-mail: pfjspartítiplaza.snuack kr \\ -Division of Nephrology, Samsung Hedical Center, Sunghymknan Lniversity School of Hedicine, Seoul 135-710, Korea \\ Received December 12, 2007
}

\begin{abstract}
Hemodialysis graft coated with paclitaxel prevents stenosis: however. large initial burst release of paclitavel causes many negative effects such as drug toxicity and inefficient drug loss. Therefore we developed and tested a novel coating method. double dipping to provide controlled and sustained release of paclitavel locally. Expanded polytetrafluoroethylene (ePTFE) grafts were dipped twice into a solution of several different paclitaxel concentrations. In vitro release tests of the double dipping method showed that early burst release could be somewhat retarded and followed by sustained release for a long time. We observed the effect of paclitaxel coating by double dipping in porcine model of arterio-venous (AV) grafts between the common carotid artery and the external jugular vein. 12 weeks after constnucting AV grafts. cross sections of the graft venous anastomosis were obtained and analyzed. Paclitavel coated ePTFE grafts by double dipping were observed to prevent neointimal hyperplasia and therefore reduced stenosis of the arteriovenous hemodialysis grafts. especially at the graft venous anastomosis sites. Our results demonstrate that second dipping of ePTFE graft. which was already coated once with paclitaxel. washes off the dnug on a surface of the graft and affects the ratio of paclitaxel on the surface to that of the inner space. possibly by diffusion: thus the early burst of dnig can be somewhat reduced.
\end{abstract}

Key Words : ePTFE, Graft, Hemodialysis, Paclitaxel, Dipping

\section{Introduction}

Currently. there are more than a million patients with end stage renal disease that are maintained on hemodialysis: these patients depend on dialysis for the removal of metabolic waste or toxic substances from the bloodstream. ${ }^{1}$ These patients require a durable access to the circulation: native arterio-venous (AV) fistulas and expanded polytetrafluoroethylene (ePTFE) grafts are the most common accesses. Graft vascular access is frequently associated with significant clinical problem such as thrombosis accompanied with stenosis of the venous anastomosis site. or of a draining vein or central vein. due to the aggressive development of venous neointimal hyperplasia. ${ }^{3}$ There have been many efforts to prevent venous stenosis or thrombosis. Dnugs such as paclitaxel (PTx) or rapanycin. eluted from stents proven to be effective in preventing neointimal hyperplasia and in-stent restenosis of the coronary artery. have been introduced to the graft or access site in a manner similar to drug-eluting stents. ${ }^{4.5}$ However fixed dnug release kinetics may' limit the effectiveness of drugs within a graft. As a result. there have been several efforts to develop a novel drug eluting graft for controlled drug release kinetics. ${ }^{6}$

The controlled diffusion of drug molecules. through a polymer matrix. is the basis of many controlled polymer release methods and devices. ${ }^{7 .}$ Several biodegradable or non-biodegradable polymers. used as a polymer matrix. have significant problems such as inflammation or tissue response. However. ePTFE which is the most common method for hemodialysis grafts has improved biocompatibility: this is because it is biologically inert. chemically and thermally stable and has excellent mechanical and anti-frictional properties. ${ }^{9-11}$ In this study. a controlled release from this biocompatible ePTFE polymer matrix is presented. It has been previously reported that paclitaxel coated by dipping and evaporation of solvent on PPTFE can adhere to the pitted polymer surface."2 Thus the drug can be released in a controlled manner because the ePTFE graft has a rough surface compared to the stainless steel bare cardiac stent. with numerous surfaces pits. However, the large burst release has been considered to be a significant problem because of the cytotoxicity of PTx ${ }^{13.14}$ In this experiment. reduction of the initial burst was achieved by a double dipping method. When a drug coated graft was dipped repeatedly into a solution with drug. partial PT $x$ in an initial burst region was washed off at the interface between graft and solution or diffused into a sustained and remained region. To examine the effect of double coating on the initial burst release. in vitro release kinetic experiments were performed. In addition. paclitaxel eluting ePTFE grafts. after double dipping. were used in surgically prepared arterio-venous grafts in a pig model to determine the affect of the coating on stenosis.

\section{Materials and Methods}

Materials. Paclitaxel was purchased from Samyang Genex Co. (South Korea). The ePTFE vascular graft was obtained from Bard Peripheral Vascular. Inc. (USA). HPLC-grade 
acetone and acetonitrile were obtained from Fischer scientific (USA) and J. T. Baker (USA) respectively. Tween 20 was purchased from Hayashi Pure Chemical Ind., Ltd. (Japan). Phosphate-buffered saline (PBS) was supplied by Cambrex (USA)

Paclitaxel Coating on an Expanded Polytetrafluoroethylene (ePTFE) Graft. Paclitaxel (Genexol ${ }^{\text {E }}$. Sanyang Genex Inc., Korea) was loaded onto ePTFE vascular grafts (IMPRA. BARD Inc.) using a dipping method. Briefly. dry paclitaxel was dissolved in acetone (Fisher Scientific) at the desired concentration and ePTFE vascular grafts were dipped vertically into these solutions and incubated for 30 minutes at $37{ }^{\circ} \mathrm{C}$. The paclitaxel-loaded ePTFE vascular grafts were then dried and maintained under vacuum overnight to remove the solvent completely. The loaded anount of the paclitaxel on the graft was measured by HPLC analysis. For double dipping. ePTFE vascular grafts were dipped vertically into the first paclitaxel solution and incubated for 30 minutes at $37^{\circ} \mathrm{C}$. The paclitaxel-loaded ePTFE vascular grafts were then dried and dipped again into the second paclitaxel solution in a same manner and incubated for about seven seconds with the proper beats to allow the grafts to soak as quickly as possible. The grafts with twice loaded paclitaxel were dried and maintained in a vacuum overnight to remove the solvent completely. Grafts were sterilized with ethylene oxide before implantation.

Morphological Characterization. The surface morphological change of the paclitaxel-coated graft was studied by scanning electron microscope (SEM) (JSM 640-A. Jeol Ltd. Tolyo. Japan). A short fragment of the graft was mounted on an aluminum stub to get a uniform layer of plate. sputter coated with about $100 \AA$ layer of platinum (Cressington 108. Jeol Ltd, Japan) and viewed on SEM.

HPLC Analysis and in vitro Release Kinetics. Drug coated grafts were soaked in the polypropylene tubes with 5 $\mathrm{mL} \mathrm{MeOH}$ and shaken for 30 minutes. The methanol solution was analyzed by HPLC (Agilent 1100 Series. USA). HPLC analysis was performed using a mobile phase of water: acetonitrile $(60: 40 \mathrm{v} / \mathrm{v})$ at a flow rate of $1.0 \mathrm{~mL} /$ min. a $4.6 \times 150 \mathrm{~mm} \mathrm{C} 18$ reverse phase column, and a UV detector set at $227.4 \mathrm{~nm}$. Under these conditions. the peak of paclitaxel was eluted at 8.07 minutes. For in vitro release studies we used a solution of phosphate-buffered saline (PBS. pH 7.4) containing 0.05\% (w/v) Tween 20 (Hayashi Pure Chemical Ind.. Ltd.. Japan), a non-ionic surfactant. as paclitaxel is poorly soluble in PBS alone. Individual ePTFE vascular grafts of $1.5 \mathrm{~cm}$ length were placed in polypropylene tubes containing $5 \mathrm{~mL}$ of release medium and incubated in a $37{ }^{\circ} \mathrm{C} / 100 \mathrm{rpm}$ hybridization incubator (FineMould Precision Ind.. Korea). At the designated times. medium was removed completely from tubes and stored for analysis for a total duration of about 16 weeks: the medium was replaced with $5 \mathrm{~mL}$ of fresh release medium. The amount of paclitaxel released into the medium was determined by HPLC.

Animals and Operative Procedure. Landrace pigs. weighing $50 \pm 5 \mathrm{~kg}$. received a single ePTFE-AV graft at both sides of the neck. between the common carotid artery' and the external jugular vein. either with or without a paclitaxel coating. The dose density of paclitaxel on the coated graft was 0.59 (range. 0.57-0.60) $\mu \mathrm{g}$ per square millimeter (in previous study), and 0.67 in the group with double dip coating. Animals were euthanized after 12 weeks. This study was reviewed and approved by the Institutional Animal Care and Use Committee (IACUC) of the Laboratory Animal Research Center at the Samsung Biomedical Research Institute (SBRI). The animal care facilities at SBRI are accre-dited by the Association for Assessment and Accreditation of Laboratory Animal Care (AAALAC) and the Institute of Laboratory Animal Resources (ILAR) and conform to the Guidelines for the Care and Use of Laboratory Animals, published by the US National Institutes of Health (NIH Publication No. 85-23. revised 1996). Before the operation and at termination, animals were fasted overnight and the induction of anesthesia was achieved with intramuscular ketamine $\mathrm{HCl}(20 \mathrm{mg} / \mathrm{kg})$ and xylazine $\mathrm{HCl}(2 \mathrm{mg} /$ $\mathrm{kg}$ ). They were then intubated and ventilated using a mixture of $\mathrm{O}_{2}$ and air (1:2) containing enflurane (2\%). An ear vein was used to continuously administer $0.1 \mathrm{mg} / \mathrm{kg}$ of vecuronium bromide. The animals were monitored using a patient monitor (M1205A Omnicare. Hewlett-Packard) and an anesthesia gas monitor (602-3A Poet IQ. Criticare system). We adopted the animal model proposed by J. I. Rotlunans $e t$ at. ${ }^{15}$ Starting at seven days preoperatively. the pigs received acetylsalicylic acid $100 \mathrm{mg} /$ day until termination. Clopidogrel (PLAVIX ${ }^{\text {B }}$ Sanofi-Synthelabo) $75 \mathrm{mg}$ was added on preoperative day one and continued at a dose of $75 \mathrm{mg} /$ day until termination. Through a longitudinal incision at the lateral side of the neck along the stemocleidomastoid muscle. the common carotid artery and the external jugular vein were exposed. Heparin $100 \mathrm{IU} / \mathrm{kg}$ was given intravenously before vessel manipulation. All AV grafts were created by experienced vascular surgeons. The artery was clamped using atraumatic clamps. and an 8-1mm arteriotony was performed. An end-to-side anastomosis was created at a $45^{\circ}$ angle using a continuous 6- 0 polypropylene suture. Reinforced, thin-walled. ringed. PTFE grafts $(6 \mathrm{~mm}$ in diameter by $15 \mathrm{~cm}$ in length) were used. These grafts were sterilized before use in ethylene oxide. The venous anastomosis was created in a similar fashion. After 12 weeks. pigs were anesthetized as described above. The proximal carotid artery supplying the graft was cannulated and ligated beyond the cannula. The grafts and adjacent vessels were perfused with saline for $3 \mathrm{~min}$. Subsequently. the grafts were perfused with formalin at physiologic pressure (i.e., $100 \mathrm{mmHg}$ ). After 2 minutes. both sides of the arteries and veins were ligated. thus allowing pressure fixation of the vessels. Grafts and adjacent vessels were then excised and immersed in formalin for at least $24 \mathrm{~h}$.

Tissue Preparation. The specimens were cut from the arterial and venous sides in 5-mum blocks and embedded in paraffin. Five $\mu \mathrm{m}$ thick sections were then prepared of veins and arteries $1 \mathrm{~cm}$ proximal and distal to the anastomosis. Serial sections were taken around the anastomosis to obtain cross sections at the center of the graft vein anastomosis. 
which were also perpendicular to flow in the vein. since stenosis of hemodialysis graft is most prominent at the juiction between the henodialysis graft and the draining vein. All sections were stained with Masson-Trichrome. These were further stained so that the intina and media could be manually traced on Masson-Trichrome stained slides which facilitated differentiation of the media.

\section{Results and Discussion}

In vitro Release Pharmacokinetics. After the paclitaxel coating, there was no significant difference observed in the ePTFE graft surface morphology before and after dipping as shown in Figure I because of the fibrous characteristics of ePTFE. In acetone solution of the PTx. the graft was wetted well and paclitaxel molecules were easily adsorbed and adhered to the textile. Thus. the morphology of drug-loaded graft was same as before drug loading because there was no physical or chemical reaction observed. Therefore, it is reasonable to use the results from in vitro testing without further physical or chemical studies. Figure 2 shows the in vito release profiles of the PTx single coated grafts containing different drug loading amounts over the same size graft in PBS-tween20 media. The amount of the drug on the graft varied from 0.388 to $0.767 \mu \mathrm{g} / \mathrm{mm}^{2}$. In grafts with higher drug ratios. initial burst release was significantly faster and was then followed by a slow release in all grafts. This result is similar to the release pattern of TAXUS. which is a paclitaxel eluting stent with a variety of ratios of PTx to

(ii)

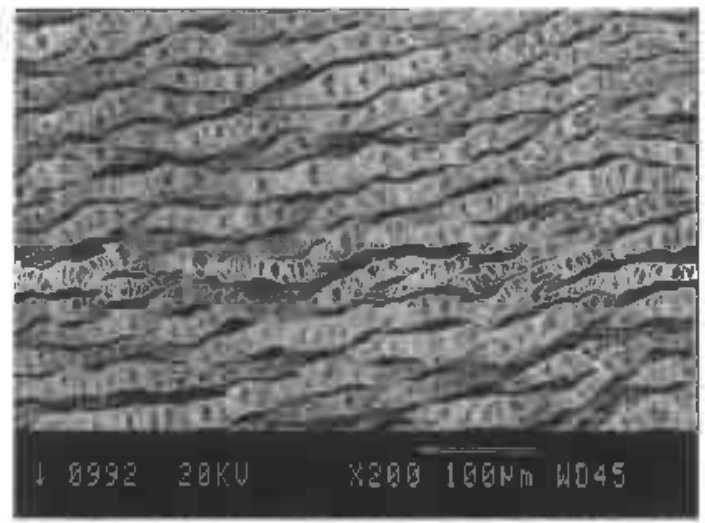

(b)

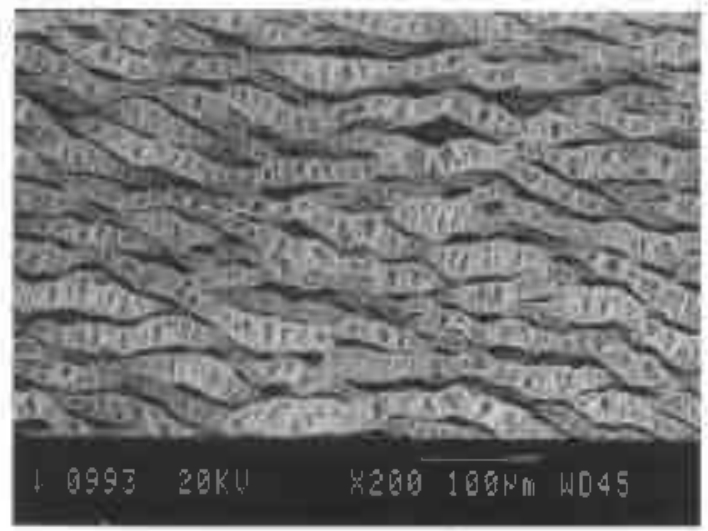

Figure 1. SEM images of ePTFE grafts betore drug coating (a) and after drug coating (b).

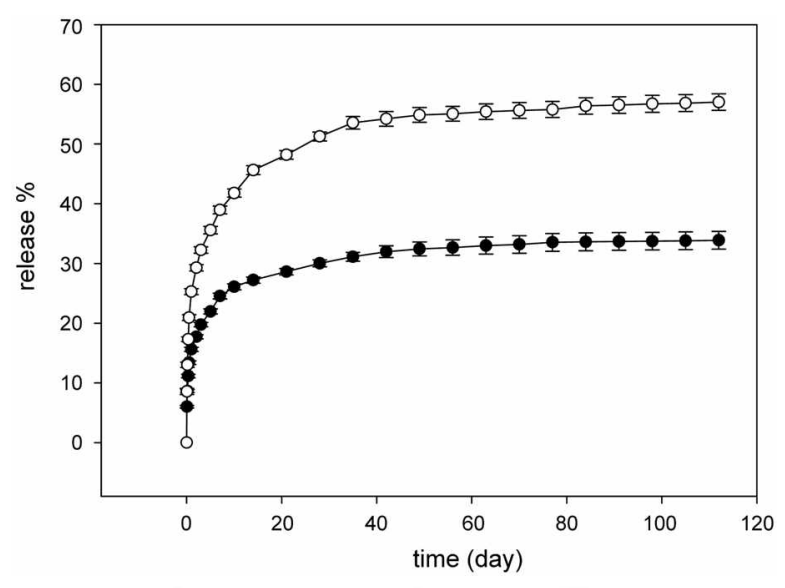

Figure 2. In vitro release \% profile of PTx from a eFTFE graft containing $0.75 \mathrm{\mu g} / \mathrm{mm}^{2}$ (open circle) and $0.38 \mu \mathrm{g} / \mathrm{mm}^{2}$ (closed circle) after single dippining. Data are an average of gratts $(\mathrm{p}<$ 0.05 , statistically signiticant .

polymer ${ }^{16,17}$ However, high concentrations of paclitaxel are locally toxic and may cause necrosis. ${ }^{18}$ In order to develop a slow and controlled release, coating experimental that varied in dipping time and number were performed. We found that the first dipping time did not affect the loading amount: however the second dipping time was very critical because of the diffusion of the first dipping drug into the second solution. To verify the effect of double coating. a drug eluting graft was incubated in PBS-tween buffer at $37^{\circ} \mathrm{C}$ with vigorous shaking. ${ }^{16}$ The different initial burst and release pattems. with the same drug loaded onto the graft, are shown in Figure 3. Paclitaxel loaded grafts using single dipping with a solution of $0.5 \mathrm{mg} / \mathrm{mL}$ contained drug of $0.388 \mu \mathrm{g} /$ $\mathrm{mm}^{2}$ and those using double coating method dipped twice into $0.3 \mathrm{mg} / \mathrm{mL}$ for a similar drug contents and contained $0.380 \mu \mathrm{g} / \mathrm{mm}^{2}$. In the case of single dipping in $1.0 \mathrm{mg} / \mathrm{mL}$ solution, for double dipping a $0.5 \mathrm{mg} / \mathrm{mL}$ paclitaxel solution was used and a total drug loading was around $0.75 \mu \mathrm{g} / \mathrm{mm}^{2}$. As shown in Figure 3, each of the grafts. coated by different coating methods (dipped once or twice) had a similar dose density of PTx in a graft but showed different release kinetics. In both grafts. the initial burst within the first 48 hours followed by a sustained release and remains. For the double dipping method (closed circle in Fig. 3), the initial burst release within one day was reduced respectively and the remaining drug amount was increased compared to the single dipping (open circle in Fig. 3). These results suggested that there are three different factors important to the release pattens of grafts: the initial burst. sustained and remained release: through second dipping. partial drug in initial burst zone is washed out and moved into the sustained and remaining zones. In this drug delivery system. the in vitro PTx release profiles (shown in Fig. 2 and 3) showed that the amounts of drug released are controlled by a diffusion system through the ePTFE polymer matrix. Therefore. the experimental data showed that double dipping into a solution allowed for a specific ratio between drug and matrix. which controls the release of dnig at different regions 
(a)

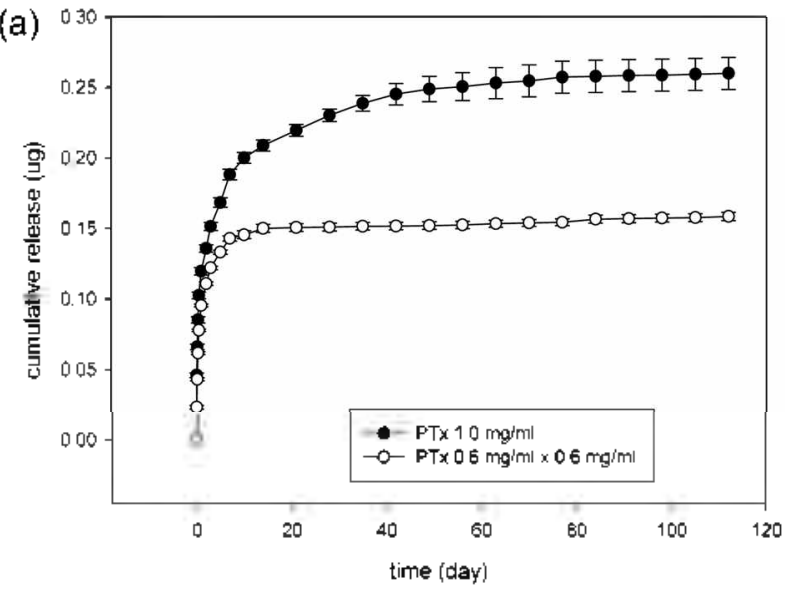

(b)

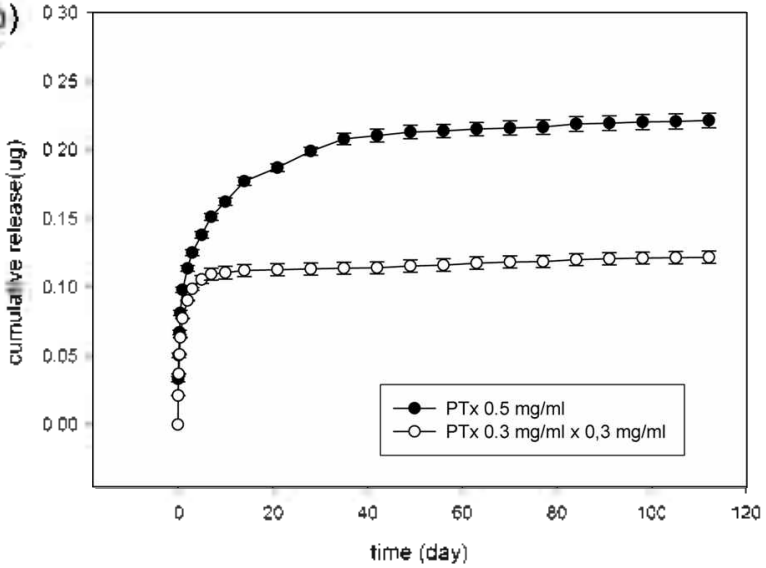

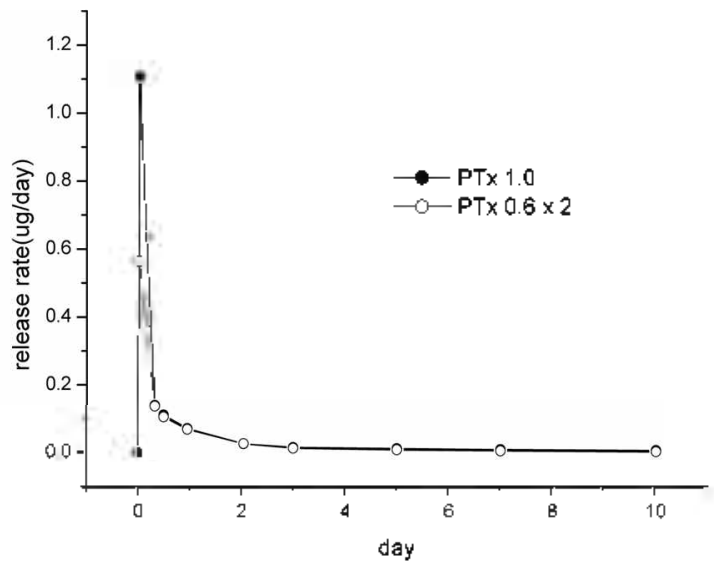

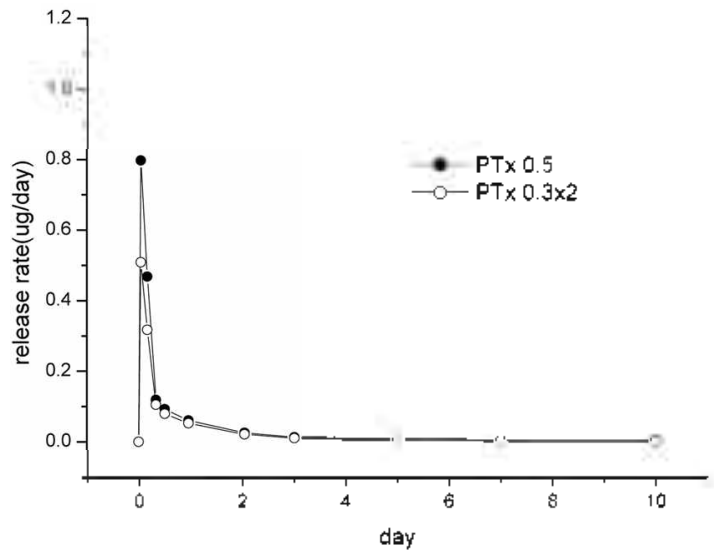

Figure 3. Drug release kinetics. Each curve represents 5 grafts $(p<0.05$ ). (a) Cumulative release and release rate of a single dipping (in 1.0 $\mathrm{mg} / \mathrm{mL}$ ) and double dipping (in $0.6 \mathrm{mg} / \mathrm{mL} \times 2$ ) with a total loading amount of about $0.75 \mathrm{tg} / \mathrm{mm}^{2}$. (b) single $(0.5 \mathrm{mg} / \mathrm{mL}$ ) vs double $(0.3 \times$ $2 \mathrm{mg} / \mathrm{mL}$ ) with about $0.4, / \mathrm{g} / \mathrm{mm}^{2}$.

of the graft. Generally. the drug release rate from a dring eluting matrix is followed by a forceful initial burst; which was the focus of this study." Like previous results for controlled dnig release ${ }^{20,21}$ we have developed a novel design for a drug eluting graft that did not cause a significant initial burst release. Our results show that the initial burst rates. using a double dipping method are controllable and flexible.

Animal Experiments and Morphometric Analysis. We recently reported that paclitaxel-coated hemodialysis grafts could reduce neointimal hyperplasia at six weeks in a porcine model ${ }^{12}$. Here. we tested whether a double dipped coated graft $(0.3 \times 2 \mathrm{mg} / \mathrm{mL}$, the drug contents on the graft were about $0.388 \mu \mathrm{g} / \mathrm{mm}^{2}$ ), which allowed a more sustained retention of drug. could inhibit neointimal hyperplasia at the venous side of the inserted graft for 12 weeks in porcine model. Four paclitaxel double coated grafts in two pigs and four control grafts in two pigs were successfully implanted
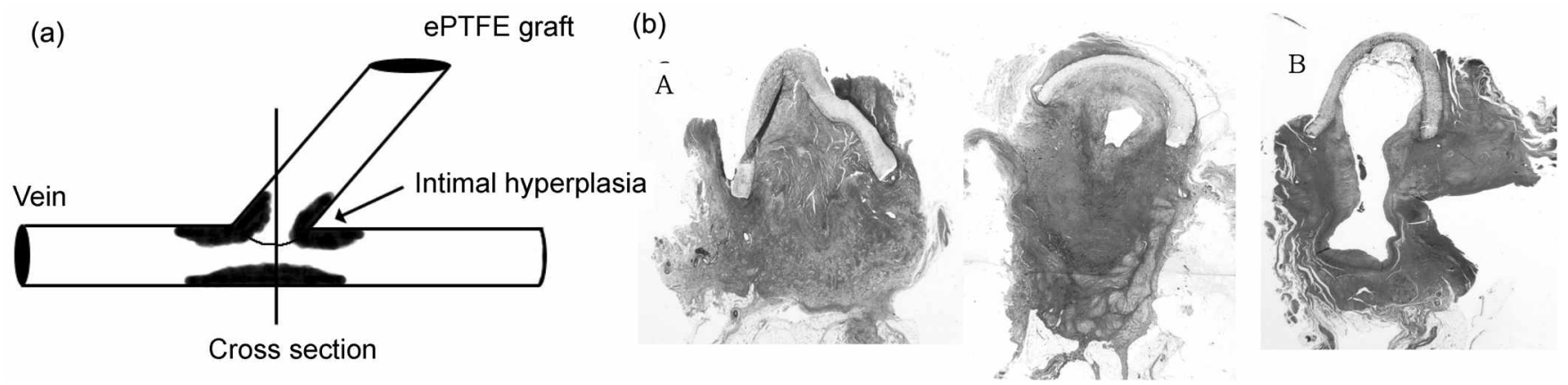

Figure 4. The cross sections of the graft venous anastomoses 12 weeks after surgery. (a) A diagram of the cross section of a venous anastomosis. Intinal hyperplasia can build up at the junction of the vein and ePTFE graft easily and at the opposite side of ePTFE graft. Cross section was made at the middle of the anastomoses. (CSVA). (b) Representative sections of venous graft anastomosis. A, control graft (without paclitaxel). B, Paclitaxel double dipping graft (with dose density of about $0.70 \mathrm{~kg} / \mathrm{mm} \mathrm{m}^{2}$ ). 
in Landrace pigs. All pigs. four PTx grafts in two pigs and four control grafts in two pigs, survived until the time of harvest at 12 weeks. The observations of cross sections from graft venous anastomosis at 12 weeks after surgery in the control and double dipped groups are shown in Figure 4. Slices of the graft were sometimes folded or detached during the process of the tissue preparation. but the location of these variations could be easily traced by considering the other adjacent sections. ${ }^{3}$ The intima was identified by a pale blue stained area inside the vascular media which appeared as layers of thick red fibers that were more evident on higher power magnification. As shown in Figure $4 \mathrm{~b}$, there was little neointima in the coated groups. Uncoated grafts showed luminal stenosis caused by intimal growth in veins and perijunctional grafts. Changes in coated grafts were minimal in sharp contrast to the controls. The intimal hyperplasia. in the arterial graft anastomosis. was minimal in both the control group and the coated group and was not further conpared. Systemic and local signs attributable to the toxic effect of the paclitaxel were not noted in the pigs in the coated group in the analy sis

\section{Conclusions}

In sunmmary. our approach for a reduced initial burst and sustained release of drug was successful. There was a significant and clear difference in release between the single and double dipping methods. This novel drug delivery system showed that controlled release of drug can be obtained by changing the number of dipping and the solvent used in a paclitaxel double coated graft without modification of physical. chentical or biological properties of the graft. In addition. our in vivo fundings showed that double coated grafts can prevent neointimal hyperplasia and the stenosis of arteriovenous hemodialysis grafts. Further experiments and clinical studies are needed to determine the release kinetics and the effects of sustained release under more realistic biological conditions.

Acknowledgement. This work was supported by the Generic Technology Development Program of Korean Ministry of Commerce. Industry, and Energy:

\section{References}

1. Kanterman. R. Y.: Vesely. T. M. J. Iasc. Interv Radiol 1995. 6(2). $267-71$.

2. Rayner. H. C.: Besarab. A.: Brown. W. W.: Disney. A.: Saito. A.: Pisoni, R. L. Am. J. Kidhey Dis. 2004, $44(5$ Suppl 2), $22-6$.

3. Melhem. M.: Kelly, B.; Zhang. J.: Kasting, G.: Li. J.: Davis, H.; Heffelfinger. S.; Desai, P.: Roy-Chaudhury, P. Blood Pwif. 2006. $2+(3) .289-98$

4. Roy-Chaudhuy. P.: Kelly. B. S.: Melhem. M.: Zhang. I.: Li. J.: Desai. P.: Munda. R.: Heffelfinger. S. C. Blood Purif. 2005. 23(1). $29-35$.

5. Axel. D. I; Kunert, W: Goggelmann. C: Oberhoff. M: Herdeg. C.: Kuttner. A.; Wild, D. H.; Brehm. B. R.: Riessen, R.: Koveker. G.: Karsch. K. R. Circulation 1997. 96(2). 636-45.

6. Cypes. S. H.: Saltzmant. W. M.: Giantnelis. E. P. J. Control. Release 2003. $90(2)$. 163-9.

7. Reinhard, C. S.; Randomsky, M. L.; Saltzman, W. M.: Hilton. J.: Brem. H. J. Controlled Release 1991, 16(3). 331-339.

8. Choy, J. H.; Son, Y. H. Bull. Korean Chem. Soc. 2004, 25(1). 122126.

9. Westedt. U.: Wittmar. M.: Hellwig. M.: Hanefeld. P:: Greiner. A.: Schaper. A. K.: Kissel. T. J. Control Release 2006. $111(1-2) .235-46$

10. Lau. K.-W.; Mak. K.-H.; Hung. J.-S.: Sigwart. U. American Heant Jounal 2004. $147(5), 764-773$

11. van der Giessen, W. J.: Lincoff, A. M.: Schwartz. R. S.: van Beusekom. H. M.: Serruys. P. W.: Holmes. D. R. Jr.: Ellis. S. G.: Topol. E. J. Circulation 1996. 94(7). 1690)-7.

12. Lee. B. H.: Nam. H. Y.: Kwon. T.: Kim. S. J.: Kwon. G. Y.: Jeon. H. J; Lim. H. J; Lee. W. K.: Park. J. S.; Ko. J. Y; Kim. D. J. Nephol Dial Transplant 2006, 21(9). 2432-8.

13. Huang. X.; Brazel, C. S. Jounal of Controlled Release 2001, 73 . $121-136$

14. Bissery. M. C.: Nolyntek. G.: Sanderink. G. I.: Lavelle. F. Anticancer Dougs 1995. 6(3). 339-55. 363-8.

15. Rotmans. J. I.: Velema, E.: Verhagen, H. J.: Blankensteijn. J. D; Kastelein, J. J.: de Kleijn. D. P.: Yo, M.; Pasterkamp, G.: Stroes, E. S. J. Swg. Res. 2003. 113(1), 161-71.

16. Sipos. L.: Som. A.: Faust. R.: Richard. R:: Schwarz. M:: Ranade. S.: Boden. M.: Chan. K. Bionacronolectles 2005. 6(5). 257(1)-82.

17. Ranade. S. V: Miller. K. M: Richard. R. E. Chan. A. K: Allen. M J.: Helmus. M. N. J. Bioned M Iater Res. A 2004, 71(4), 625-34.

18. Ganansia-Levmarie. V:; Bischoff. P.: Bergerat. J. P: Holl, V. Cum: Hed Chem Anicancer Agents 2003. 3(4), 291-306.

19. Borgquist. P.: Koner. A.: Piculell. L.: Larsson. A.: Axelsson1. A. J. Control. Release 2006. 113(3). $216-25$.

20. Lim. H. J.: Nam. H. Y.: Lee. B. H.: Kim. D. ..: Ko. .. Y.: Park. J. s. Biotechol. Prog 2007. 23(3), 693-697.

21. Nam. S. H; Nam, H. Y.: Joo. J. R; Baek, I. S.: Park. J.-s. Bull. Korean Chem. Soc. 2007. 28(3), 397-4(12. 